Forecasting stock market return with nonlinearity: a genetic programming approach

Shusheng Ding, Tianxiang Cui, Xihan Xiong \& Ruibin Bai

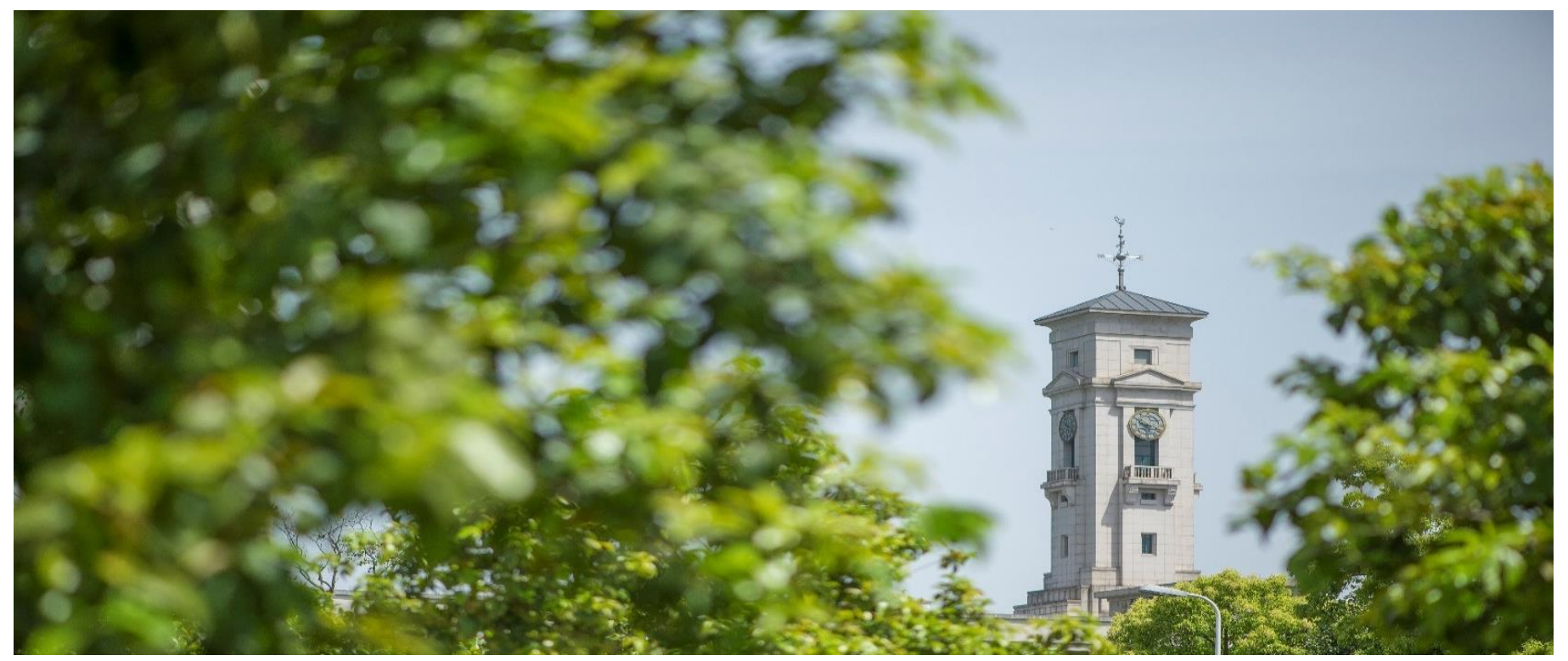


University of Nottingham Ningbo China, 199 Taikang East Road, Ningbo, 315100, Zhejiang, China.

First published 2020

This work is made available under the terms of the Creative Commons Attribution 4.0 International License:

http://creativecommons.org/licenses/by/4.0

The work is licenced to the University of Nottingham Ningbo China under the Global University Publication Licence:

https://www.nottingham.edu.cn/en/library/documents/researchsupport/global-university-publications-licence.pdf 


\title{
Forecasting Stock Market Return with Nonlinearity: A Genetic Programming Approach
}

\author{
Shusheng Ding · Tianxiang Cui · Xihan \\ Xiong • Ruibin Bai
}

Received: date / Accepted: date

\begin{abstract}
The issue whether return in the stock market is predictable remains ambiguous. This paper attempts to establish new return forecasting models in order to contribute on addressing this issue. In contrast to existing literatures, we first reveal that the model forecasting accuracy can be improved through better model specification without adding any new variables. Instead of having a unified return forecasting model, we argue that stock markets in different countries shall have different forecasting models. Furthermore, we adopt an evolutionary procedure called Genetic Programming (GP), to develop our new models with nonlinearity. Our newly-developed forecasting models are testified to be more accurate than traditional AR-family models. More importantly, the trading strategy we propose based on our forecasting models has been verified to be highly profitable in different types of stock markets in terms of stock index futures trading.
\end{abstract}

Keywords return forecasting $\cdot$ nonlinear models $\cdot$ genetic programming

\section{Introduction}

A crucial question for open discussions in finance is whether future stock returns are predictable (see Fama 1970), and this issue is also controversial (e.g. Ang and Bekaert 2006). A plethora of studies (such as Fama and French 1988;

Shusheng Ding

School of Business, Ningbo University

Tianxiang Cui

School of Computer Science, The University of Nottingham Ningbo China

E-mail: zx10551@nottingham.edu.cn

Xihan Xiong

Department of Mathematics, The London School of Economics and Political Science

Ruibin Bai

School of Computer Science, The University of Nottingham Ningbo China 
Campbell and Yogo 2006; Bollerslev et al. 2015; Goleza and Koudijs 2018; Liu et al. 2019) have shown that the stock returns are predictable by using relevant variables, such as dividend. On the other hand, however, many others remain skeptical about the stock return predictability (Welch and Goyal 2007).

\subsection{Research Background and Contributions}

This paper aims to further examine this issue by building new return forecasting models using the genetic programming approach. Contributions of our paper stem from several aspects. Firstly, we unveil that the model forecasting accuracy can be improved through better model specification without adding any new variables. Seeking the relevant variables for forecasting future return$\mathrm{s}$ has been witnessed in burgeoning literatures (see Fama and French 1988; Nelson and Kim 1993; Campbell and Shiller 1988). In these aforementioned works, they only focus on demonstrating the potential of different variables in forecasting stock market returns. Nevertheless, works dedicated to calibrate model specifications are scant. As a result, in this paper, in contrast to existing literatures, we only use lagged market return as the future return predictors. We do not add any new variable to our model because we intend to show that new model specification can improve the model prediction power. It might be complementary to existing literatures that better model specification could be equivalently vital as including new variables, which reinforces predictive power of return forecasting model.

Furthermore, we adopt an evolutionary procedure, namely Genetic Programming (GP), to develop our new models with nonlinearity. The nonlinear dependence of the return time series has been well documented (see Scheinkman and LeBaron 1989; Ding et al. 1993), especially for those emerging markets (see Avdoulas et al. 2018). We use GP to search the potential forms of the return forecasting model using only the lagged returns as predictors. GP is a specialized form of Evolutionary Algorithm (EA) inspired by Darwin's theory of evolution. The basic idea behind is to simulate the survival of the fittest principle in a biological category, such that the favoured race of the successive generations will be naturally chosen for preservation. A distinct feature of GP compared to other evolutionary methods is the tree structure which gives not only an optimised solution but also the solution method.

More importantly, discontinuous movements like jumps happen frequently in the stock market and traditional return forecasting model is difficult to capture such discontinuity (see Kim and Mei 2001; Chan and Maheu 2002; Cremers et al. 2015). The jump process in terms of natural log function is usually used to approximate price discontinuous movements in the futures and options markets, and this formulation tends to be nonlinear (Bates 1996; Kou 2002). Therefore, we also include the natural log function in our GP framework in order to capture such discontinuity and it would be advantageous to use GP as the solution model provided by GP is normally nonlinear because of its 
evolutionary nature. Therefore, the accuracy of GP model specification might be heightened by capturing such nonlinearity.

Besides, stock markets in different countries might exhibit distinguishing characteristics. It is arguable that the characteristics in emerging markets could be entirely contrasting to those in developed markets. Therefore, we classify markets into subgroups and we employ different model specifications for different market types. Like (Gencay and Selcuk 2004) show that different countries have different moment properties at right and left tails of their return distributions, which may entail different risk-reward relationship. (Lee et al. 2015) illustrate that empirical evidence from the U.S. stock market might be quite distinctive to other countries, especially Asian countries, under the structural VAR model. It is also well documented that different stock markets have different characteristics in the literature (see Chen et al. 2006; Choudhry and Garg 2008). Consequently, we argue that different countries shall have different return forecasting models that suit their own countries' characteristics. Basically, developed countries and emerging countries shall be categorized into two different types of economics, which shall have different return forecasting models. Furthermore, during different time periods, stock markets may also exhibit different features. Therefore, dynamic model specification with GP could well be overwhelmingly favourable compared with static models even with new variables.

Therefore, the goal of the paper is to adopt GP to generate the best models that can predict future stock returns without adding any other variables but with high accuracy. We categorize different countries into different groups and we have developed an appropriate model for each group. More importantly, our proposed models are more accurate in predicting returns and can be used to develop corresponding trading strategies with high profitability. The trading strategy is used in the stock index futures market. It is noticeable that futures trading differs from stock trading. Specifically, futures contract has maturity, which indicates that futures contract has an expired date and all futures positions would be closed on that day automatically. Therefore, we use our model to forecast one-day ahead return and implement the intra-day trading strategy. In other words, our futures position would be opened and closed on the same date. Additionally, unlike stock investment, investors can earn money even if the market return is negative because they can take short positions of stock index futures. As a result, the traditional stock trading strategy like buy and hold would be irrelevant and thus it might be inappropriate to use such strategy as a benchmark. Therefore, we adopt the same trading strategy for all tested models. Compared with other AR-family models with same trading strategy in futures market, our model exhibits $55 \%$ profitability on average while other models only have $40 \%$.

Empirically, we are able to demonstrate that our models have superior performance in forecasting future returns compared with AutoRegressive (AR) family models in both linear and nonlinear forms. The improvement rate is around $30 \%$ for in-sample fitting and around $40 \%$ for out-of-sample forecasting. Furthermore, we have exploited a trading strategy based on our models. 
The profitability of our trading strategy is around $20 \%$ for developed markets and around $60 \%$ for emerging markets from 2012 to 2017, which are noticeably higher than traditional AR family models. Moreover, we also adopt a traditional non-linear model for the robustness check and our models outperforms the traditional non-linear model, which verifies the robustness of our results.

\subsection{Literature Overview}

Predicting future returns with relevant variables has been the focal point in literatures. Dividend is the most popular variable for predicting future stock returns among those works. (Fama and French 1988, Nelson and Kim 1993 and Campbell and Shiller 1988) show that the capability of dividend conveying the future dividend growth and expected return information may yield the successful cases of stock return prediction. The Price/Earnings $(\mathrm{P} / \mathrm{E})$ ratio has also been well documented in the return predictability literatures. Like (Lamont 1998) maintains that the $\mathrm{P} / \mathrm{E}$ ratio holds the predictive power to predict the future stock returns in addition to dividend. Moreover, Book/Market (B/M) ratio also plays an important role in stock predictability research. (Jiang and Lee 2007) demonstrate the prediction power of $\mathrm{B} / \mathrm{M}$ ratio and log dividend yield in terms of return forecasting performance. (Aydogan and Gursoy 2000), unfold the fact that $\mathrm{P} / \mathrm{E}$ as well as $\mathrm{B} / \mathrm{M}$ ratios carry the ability of predicting future returns, especially over long time periods. More recently, (Cremers and Weinbaum 2010) use deviations from put-call parity to predict future stock returns. However, the prediction power of those models is quite limited as (Ang and Bekaert 2006) argue that return forecasting model with dividend fails to exhibit any long-horizon predictive power. More importantly, forecasting models based on the dividend and earnings yield may also have instability problems (see Lettau and Ludvigson 2001; Goyal and Welch 2003; Paye and Timmermann 2006; Cai et al. 2015). Moreover, since our paper also focuses on developing trading strategies, the technical analysis paper such as (Park and Irwin 2007, Batten et al. 2018 and Jiang et al. 2019) as well as the recent non-linear model works such as (Zhao et al. 2019 and Facchini et al. 2020) could also be relevant.

GP also holds the elegant characteristics that one can build the relevant performance criterion directly into the search procedure. Furthermore, it has been shown that GP has been adopted in various financial areas. For example, (Manahov et al. 2015) has utilized a Strongly Typed Genetic Programming (STGP) based trading algorithm to forecast one-day-ahead stock return. The STGP-based system enables them to investigate the stock return forecasting through groups of artificial traders. They find that the STGP-based forecasting results dominate other benchmark forecasts in a short time horizon. (Pimenta et al. 2017) apply genetic programming with multiobjective optimization to develop an automated investing method and this method is proven to be quite profitable in the Brazil stock exchange market (BOVESPA). More recently, the applications of GP have been also witnessed in different research fields 
other than finance (see Bhola et al. 2019; Chen and Gao 2019; Shoba and Rajavel 2020). Therefore, we utilize GP to build our new return forecasting models with nonlinearity and embedded nonlinearity characteristics in our models could enhance the model performance in predicting future returns.

Therefore, developing new return forecasting model without adding new variables is essential since less variable might make model more stable. Further, model specification with features like nonlinearity would be also helpful and GP method would be favorable.

\subsection{Paper Structure}

The remainder of the paper is organized as follows. Section 2 gives the detailed information about the data and methodology we use. Section 3 describes the GP algorithm. Section 4 shows the empirical return forecasting results. Section 5 presents the empirical results of the trading strategy based on our return forecasting models. Section 6 concludes our paper.

\section{Data and Methodology}

\subsection{The Data}

We obtain four countries' stock index from WIND database with daily frequency, from January 1, 2006 to December 31 2017. The full sample constituted by four countries contains two subsamples, which are developed economics and emerging economics. For developed economics, we use S\&P 500 index of US and Nikkei 225 index of Japan. For emerging economics, we use Sensex 30 index of India and CSI 300 index of China. As pointed out in (Batten et al. 2018), the sample composition could have an impact on the model performance. In order to maintain the in-sample and out-of-sample periods have the same observations, we divide the sample from 2006-2011 and 2012-2017. This can help models to show their performance in a relatively similar way in both in-sample and out-of-sample periods and assist us to enhance our model performance in the out-of-sample period. For the in-sample test, we use the full sample period, which means the input data and the forecasted returns will be both the whole sample period. For the out-of-sample period, we use January 1, 2006 to December 31, 2011 as the estimation period and January 1, 2012 to December 312017 as the forecasting period, which means we use the period January 1, 2006 to December 31, 2011 as the input data to forecast the stock return of the period January 1, 2012 to December 31 2017. Specifically, in-sample test indicates that we use available data to forecast values within the estimation period while out-of-sample test means we use available data to forecast values outside the estimation period. For the trading strategy empirical test, we also use the corresponding stock index futures data for the four stock markets with the period from January 1, 2012 to December 31 2017. In addition, for both 
in-sample and out-of-sample tests, we use 1-day ahead prediction during the data period and the statistical test for error differences will be also employed.

\subsection{Model Specifications and Variable Estimation}

The main variable we use in this paper is the return, which can be defined as (Andersen and Bollerslev 1998):

$$
r_{t}=\ln \left(\frac{P_{t}}{P_{t-1}}\right)
$$

where $P_{t}$ is the spot price of a stock or a stock index.

Table 1 summarizes the detailed statistics of stock index returns for four countries.

\begin{tabular}{llllll}
\hline Variable & Obs & Mean & Std. Dev. & Min & Max \\
\hline$r_{U S}$ & 3,020 & 0.00025 & 0.0122 & -0.094 & 0.109 \\
$r_{\text {Japan }}$ & 2,957 & 0.00012 & 0.0155 & -0.121 & 0.132 \\
$r_{\text {China }}$ & 2,916 & 0.0005 & 0.0198 & -0.118 & 0.134 \\
$r_{\text {India }}$ & 2,928 & 0.0004 & 0.0146 & -0.116 & 0.159 \\
\hline
\end{tabular}

Table 1 Statistical summary of variables used for the 12-year returns of four countries.

The AR-class model has been widely used in the financial literatures for return forecasting (see Ferrara et al. 2015; Avdoulas et al. 2018). We use the AR-class model to forecast stock index returns, with both linear and nonlinear specifications as benchmark models. For the linear benchmark model, we use the standard Autoregressive (AR) model. For the non-linear benchmark models, we use the SETAR (Self-Exciting Threshold Autoregression) model and the STAR (Smooth Transition Autoregressive) model. Both of the models are time series models, which assume that data order is in time sequence. For the linear ARMA model, it assumes a linear relationship between past asset returns and future asset returns. In other words, future asset returns can be envisioned as a function of past asset returns with linear combination. On the other hand, the nonlinear models such as SETAR and STAR models, they assume a nonlinear relationship between past asset returns and future asset returns. Those models can be viewed as structural models where they use thresholds to distinguish returns in different structures.

Then, we provide a brief description of the models implemented in our analysis, (Terasvirta 1994; Hurn et al. 2016). The benchmark forecasting linear autoregressive model (AR) of order $p$ ( $p$ is the number of lagged autorregressive term $y_{t}$ ), for a given horizon $h$ :

$$
y_{t+h}=\alpha+\boldsymbol{\beta} \boldsymbol{X}_{\boldsymbol{t}}+\varepsilon_{t+h}
$$

where $\mathbf{X}_{t}=\left(y_{t}, y_{t-1}, \ldots, y_{t-p+1}\right), \varepsilon_{t+h}$ has a finite variance $\sigma_{\varepsilon}^{2}, \alpha$ is a constant, and $\boldsymbol{\beta}$ is a p-vector of parameters. Specific model is selected by Bayesian 
Information Criterion (BIC). The BIC is mathematically defined as:

$$
k \ln (n)-2 \ln (L(\theta)),
$$

where $n$ is the data size, $k$ is the number of parameters estimated, $\theta$ is the set of all parameters, in particular, $L(\theta)$ represents the maximized value of the likelihood function for the estimated model with $\theta$.

We need the maximum value of $L(\theta)$, the lowest BIC is thereby preferred. Moreover, parameters are estimated by Ordinary Least Squares (OLS) linear regression method. OLS linear regression method uncovers the parameters of a linear function consisting a number of independent variables by minimizing the sum of the squares of the differences between the observed dependent variable and predicted dependent variable (calculated through the linear function with independent variables). As has been documented in the literature (Marcellino et al. 2006), the forecasting model in Equation (1) often outperforms alternative and more sophisticated univariate and multivariate models. In this work, we focus on three classes of well-known autoregressive models that nest the AR $(p)$ model in Equation (1), namely ARMA model, TAR model and STAR model.

In additional to the traditional ARMA model, we use two nonlinear models, TAR and STAR. The Threshold Autoregressive (TAR) model was developed by (Tong 1978), which assumes that the regime-switching that occurs at time t can be determined by an observable variable $q$ relative to a threshold value, denoted by $c$. The model presumes that the time series may behave differently corresponding to different regimes where the regime-switching point depends on the past values of the time series and the specific threshold value $c$. A specific case of TAR model is a SETAR (Self-Exciting Threshold Autoregression) model, which assumes that the threshold variable $q$ can be selected to be the lagged value of the time series itself (Tong 1990; Hansen 1997; Hansen 2000). The most general case is to presume that the model has two regimes to switch, where the specific model for order $p$ can be defined as:

$$
y_{t}=\left(\boldsymbol{\alpha}_{\mathbf{1}}+\boldsymbol{\beta}_{\mathbf{1}} \boldsymbol{X}_{\boldsymbol{t}}\right) I\left(y_{t-q} \leq c\right)+\left(\boldsymbol{\alpha}_{\mathbf{2}}+\boldsymbol{\beta}_{\mathbf{2}} \boldsymbol{X}_{\boldsymbol{t}}\right) I\left(y_{t-q}>c\right)+\varepsilon_{\boldsymbol{t}}
$$

where I $[\mathrm{A}]$ is an indicator function with $\mathrm{I}(\mathrm{A})=1$ if the event A occurs and I $(\mathrm{A})=0$ otherwise; $\boldsymbol{X}_{\boldsymbol{t}}=\left(1, y_{t-1}, y_{t-2}, \ldots, y_{t-p}\right) ; \boldsymbol{\alpha}_{\mathbf{1}}+\boldsymbol{\beta}_{\mathbf{1}}$ and $\boldsymbol{\alpha}_{\mathbf{2}}+\boldsymbol{\beta}_{\mathbf{2}}$ are $\mathrm{p}$ +1 -vectors of parameters.

Besides, we also use the STAR model and the most general case of the STAR model for order $p$ can be expressed by the following function formula:

$$
y_{t+h}=\left(\boldsymbol{\alpha}_{\mathbf{1}}+\boldsymbol{\beta}_{\mathbf{1}} \boldsymbol{X}_{\boldsymbol{t}}\right)\left(1-G\left(z_{t-d}, \gamma, c\right)+\left(\boldsymbol{\alpha}_{\mathbf{2}}+\boldsymbol{\beta}_{\mathbf{2}} \boldsymbol{X}_{\boldsymbol{t}}\right) G\left(z_{t-d}, \gamma, c\right)+\boldsymbol{\varepsilon}_{\boldsymbol{t}+\boldsymbol{h}}\right.
$$

where $\boldsymbol{X}_{\boldsymbol{t}}=\left(y_{t}, y_{t-1}, y_{t-2}, \ldots, y_{t-p+1}\right) ; \boldsymbol{\alpha}_{\mathbf{1}}+\boldsymbol{\beta}_{\mathbf{1}}$ and $\boldsymbol{\alpha}_{\mathbf{2}}+\boldsymbol{\beta}_{\mathbf{2}}$ are p +1 -vectors of parameters. $G(\cdot)$ is the smooth-transition function.

Specifically, the smooth transition function can be determined by one of the following functions: 
one is a logistic function, which is:

$$
G\left(z_{t-d}, \gamma, c\right)=\left[1+\exp \left(-\frac{\gamma}{\sigma_{z_{t-d}}}\left(z_{t-d}-c\right)\right]^{-1}\right.
$$

or an exponential function, which is:

$$
G\left(z_{t-d}, \gamma, c\right)=\left[1+\exp \left(-\frac{\gamma}{\sigma_{z_{t-d}}}\left(z_{t-d}-c\right)^{2}\right]\right.
$$

where $\gamma$ is the smoothing parameter that controls for the shape of regime changes; $z_{t-d}$ is the transition variable, $\sigma_{z_{t-d}}$ is the standard deviation of the transition variable and $c$ is the threshold parameter.

\section{Model Evolution Using Genetic Programming}

\subsection{Preliminaries}

In this section, we will develop our return forecasting model based on the estimated variables in section 2. For the specific model development, we will adopt an evolutionary search method, Genetic Programming (GP). GP is an evolutionary computation (EC) technique inspired by biological process (see Banzhaf et al. 1998; Hirsh et al. 2000; Poli et al. 2008). Since the form of return forecasting model with nonlinearity is uncertain, it would be beneficial to adopt GP method. One big advantage of adopting GP in this work is that it can allow one to be agnostic about the general form of the model. In GP, a population of computer programs is evolved based on the principles of natural selection originated from Darwin's theory of evolution. After certain number of generations, GP can transform populations of programs into new and better programs. As stated in (Poli et al. 2008), GP has been very successful at evolving novel and unexpected ways of solving problems.

The main idea of our GP approach is as follows: it firstly generates a random population of functions, and then it evaluates the quality of each individual function, which is the difference between the generated function and the targeted function ( $r_{t}$ in this work, see 3.2 for details). Such quality is usually called the fitness of the individual. Next, one or two function(s) will be probabilistically selected based on its fitness in order to participate in the genetic operations. Normally there are two genetic operations, one is called crossover and another is called mutation. The crossover operation is used to create a new child function (called offspring) by randomly choosing some subitems from two selected functions (called parents, which are usually polynomials) and recombining the subitems from the two functions together. The mutation operation is used to create a new child function by choosing some random subitems from one selected function and altering them. After new individuals are created, their fitness will be calculated again, and genetic operations will also be performed again to evaluate the newly-generated function. The genetic operations will be undertaken under the probability of crossover and mutation, which will 
be outlined later. This whole process is mainly based on the aforementioned principles of evolution and will be repeated until an acceptable solution is found or other termination criterion is satisfied (usually up to some certain number of generations). The best individual will be returned as the solution, which is effectively the new return forecasting model.

\subsection{Genetic Programming System}

For our model development, we reduce the forecasting task to the computation of the following function based on our GP approach using the data sample period from January 1, 2006 to December 31, 2017:

$$
f\left(r_{t-1}, r_{t-2}, r_{t-3}\right)=r_{t}
$$

where $r_{t-1}, r_{t-2}, r_{t-3}$ are the lagged terms of the stock index return. Our goal is to find the most relevant terms that have effects on predicting the future stock index return.

Our GP approach consists of the following parts:

- Terminal Set: $r_{t-1}, r_{t-2}, r_{t-3}$.

- Function Set:,,$+- \times, \ln I(\cdot)$ (the indicator function).

- Fitness measure: the error between the value of the individual function and the corresponding desired output (i.e. $r_{t}$ ).

- GP parameters: population $=10000$, the maximum length of the program $=1000$ (i.e. up to 1000 subitems within one polynomial function), probability of crossover operation $=0.8$ (i.e. $80 \%$ of population functions will be mixed with other functions to generate new functions) and probability of mutation operation $=0.1$ (i.e. $10 \%$ of population functions will be mutated to generate new functions).

- Termination criterion: when the fitness measure reaches 0 or the system runs up to 100 generations, the system will terminate (For our work, the fitness measure will never reach 0 , therefore the system will terminate after 100 generations).

The general procedure of our GP approach can be found in Algorithm 1.

\subsection{Model Development}

In order to enhance the accuracy of the developed model, we categorize our sample into two subsamples, namely developed economics (including US and Japan) and emerging economics (including China and India) and we run the two subsamples separately. With the settings stated in the previous section, we ran our GP algorithm for 50 times for each subsample. After simplification, the best function we obtained is the following model for the developed economics:

$r_{t}=r_{t-2}+r_{t-3}+r_{t-1}^{2}+\ln \left(r_{t-2} * r_{t-3}\right) I\left(r_{t-2} * r_{t-3}>0\right)+\ln \left(r_{t-3}^{2}\right)+\ln \left(r_{t-3}^{3}\right) I\left(r_{t-3}>0\right)$ 


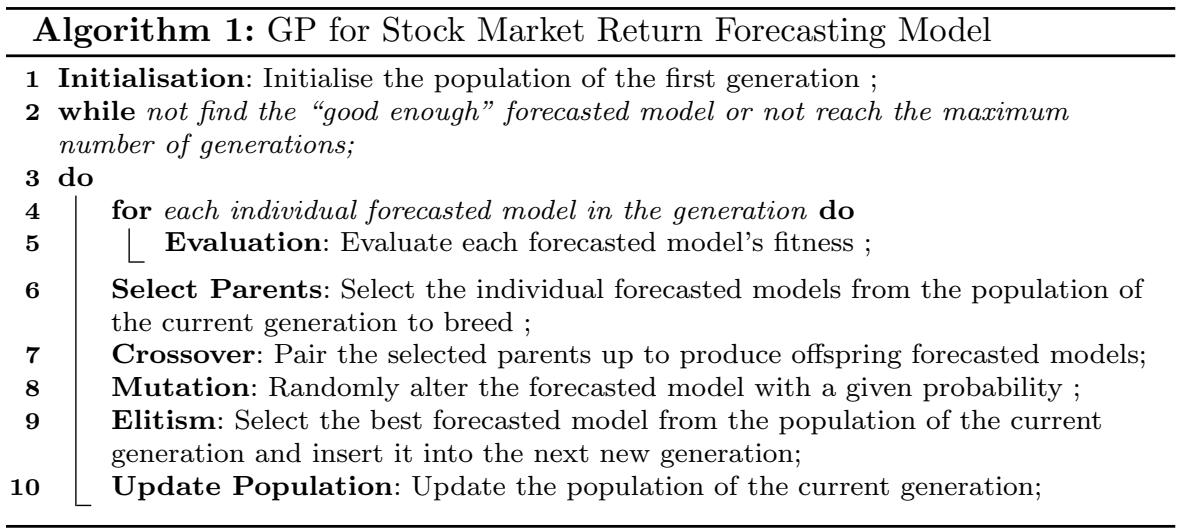

where $r_{t-q}$ is the lagged term of return and $\mathrm{I}$ is the indicator function: $\mathrm{I}=1$ if the condition in the parenthesis holds and $\mathrm{I}=0$ otherwise.

We denote this model as the Nonlinear Return Forecasting Model 1 (NRFM1).

The best function we obtained is the following model for emerging economics:

$$
r_{t}=r_{t-2}+r_{t-3}+r_{t-1}^{2}+r_{t-2}^{2}+r_{t-3}^{2}+r_{t-2} r_{t-3}
$$

where $r_{t-q}$ is the lagged term of return.

We denote this model as the Nonlinear Return Forecasting Model 2 (NRFM2).

These two newly-developed models display distinctive components. For NRFM1, it has three natural log items whereas NRFM2 has none. The natural log items might be a nexus of jumps embedded in the stock price process. Consider the following jump-diffusion stochastic differential equation (SDE) that depicts a stock process with log-normal distribution:

$$
d[\ln (S(t))]=\mu_{d} d t+\sigma_{d} d Z(t)+\ln (1+J(Q)) d P(t),
$$

where $\mu_{d}$ is the log-diffusion drift, $\sigma_{d}$ is the volatility of the stock return and $\ln (1+J(Q))$ is the log-return jump-amplitude with the a simple Poisson jump process $d P(t)$ with jump rate $\lambda$ and the process ensures that $J(Q)>-1$.

Therefore, it is comparable that the natural log items in our model are analogous to the jump function in the SDE. More importantly, the indicator function is analogous to the Poisson jump process because the Poisson jump process occurs at a predetermined rate $\lambda$ while our indictor function implies the strong serial correlation of returns, which may also be interpreted as the jump occurring probability. When the returns are positively related, for example, returns are all positive or negative during a couple of days, then the jump is more likely to happen. Therefore, the NRFM1 may capture the jump ingredient in the developed markets by comprising those natural log items.

On the other hand, however, there is no natural log item in NRFM2 for emerging markets. Accordingly, NRFM2 unfolds the fact that jumps are less 
likely to occur in emerging markets. The reason is that returns in chosen emerging markets are more bounded. For instance, there is a price limit system in the Chinese stock market, which binds the daily return within $\pm 10 \%$. Similarly, in the Indian stock market, the price movement has also been constrained. In particular, Bombay Stock Exchange (BSE) has implemented circuit filters system and set the trigger of circuit filters at $10 \%$ (rise or fall). Those binding regulations prominently reduce the jump probabilities in both emerging markets. As a result, we acquire two models with distinguishing features that can represent different types of markets.

\section{Empirical Results of Return Forecasting}

This section gives both empirical results for regression models and model performance of return forecasting. In particular, we compare our data fitting results as well as prediction results with three ARMA models, namely, ARMA, SETAR and STAR as well as a High Moments Return Forecasting Model (HMRFM). For the model performance evaluation, we use Mean Absolute Error (MAE) for the model accuracy test. The periodic averaged MAE can be defined as:

$$
M A E_{T}=\frac{1}{T} \sum_{t=1}^{T} \mid \text { Observed }_{t}-\text { Predicted }_{t} \mid
$$

where $\mathrm{T}$ represents the number of observations embedded in the forecast-

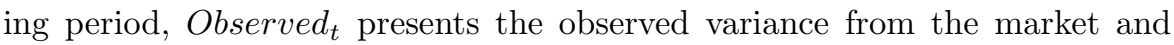
Predicted $_{t}$ presents the variance predicted from the models.

For the robustness purpose, we also use Mean Squared Error (MSE) to measure the model performance for both in-sample fitting and out-of-sample forecasting tests since our daily data could be quite noisy (Pong et al. 2004; Golosnoy et al. 2014; Bollerslev et al. 2016). The periodic averaged MSE can be defined as:

$$
M S E_{T}=\frac{1}{T} \sum_{t=1}^{T}\left(\text { Observed }_{t}-\text { Predicted }_{t}\right)^{2},
$$

where $\mathrm{T}$ represents the number of observations embedded in the forecast-

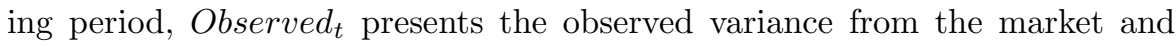
Predicted $_{t}$ presents the variance predicted from the models.

Lower MSE indicates higher forecasting accuracy.

For the ARMA model estimation, we use the AIC (Akaike Information Criteria) and BIC (Bayesian Information Criterion) to determine the optimal lag. Specifically, we use ARMA $(1,1)$ for Japan and India and ARMA $(2,2)$ for China and US regarding the in-sample test and we use $\operatorname{ARMA}(1,1)$ for Japan and ARMA $(2,2)$ for China, US and India regarding the out-of-sample test. 


\subsection{In-sample Data Fitting}

For the in-sample modeling, we compare three AR-class models with our models in fitting future stock market returns. In particular, we use the NRFM1 to forecast the stock returns of US and Japan, and we use the NRFM2 to forecast the stock returns of China and India. Table 2 shows the in-sample fitting MAE against ARMA, SETAR and STAR models. In general, our models outperform other three models with the improvement rate averaged around $25 \%$. For the NRFM1 model performs better in predicting the stock return for developed countries than NRFM2 for emerging markets (see Table 2 and Table 3). It might because that the developed market prices reflect more information than emerging markets, whose market efficiency tend to be low. This result is in accord with existing documents that most Asian markets display weak or no market efficiency (Kim and Shamsuddin 2008). Less reflected information in the market prices dilute the accuracy of data fitting within the sample by using only market price as the predicting variable. It also explains the reason that our model performs better in predicting the stock return for US than for Japan.

\begin{tabular}{|c|c|c|c|c|c|c|c|c|}
\hline & \multicolumn{2}{|c|}{ ARMA NRFM1 Improve } & \multicolumn{3}{|c|}{ SETAR NRFM1 Improve } & \multicolumn{3}{|c|}{ LSTAR NRFM1 Improve } \\
\hline & (MAE) (MAE) & Rate (p-value) & (MAE) & (MAE) & Rate (p-value & (MAE) & (MAE) & Rate (p-value) \\
\hline \multicolumn{9}{|l|}{ Country } \\
\hline 然 & 0.0077 & 6 & 0.0076 & 0.0 & & 0.0078 & 0.0029 & $0.00)$ \\
\hline \multirow[t]{3}{*}{ Japan } & $0.0107 \quad 0.0088$ & 17.8 & 0.0106 & 0.0088 & 17 . & 0.0106 & 0.0088 & $17.0 \%(0.00)$ \\
\hline & ARMA NRFM & $\operatorname{Imp}$ & SETAF & NRFM & $2 \operatorname{Iml}$ & LSTA & NRFM & $2 \mathrm{Im}$ \\
\hline & (MAE) (MAE) & Rate (p-value) & (MAE) & (MAE) & Rate (p-value & (MAE) & (MAE) & Rate (p-value) \\
\hline China & $0.0135 \quad 0.012$ & $11.1 \%(0.00)$ & 0.014 & 0.012 & $14.2 \%(0.00)$ & 0.014 & 0.012 & $14.2 \%(0.00)$ \\
\hline India & $0.0099 \quad 0.0092$ & 7.07 & 0.0097 & 0.0092 & $6(0.12)$ & 0.0096 & 0.0092 & $4.16 \%(0.15)$ \\
\hline Average & $\begin{array}{ll}0.0104 & 0.00082 \\
\end{array}$ & $24.56 \%$ & 0.0104 & 0.0082 & $24.53 \%$ & 0.0104 & 0.0082 & $24.54 \%$ \\
\hline
\end{tabular}

Table 2 MAE of in sample fitting of stock index returns. This table presents the in-sample fitting results of four countries' stock index return forecasting of four models using the Mean Absolute Error (MAE). The p-values for statistical differences of the forecasting errors are also presented. Our models outperform all other three models.

\subsection{Out-of-sample Forecasting}

On the other hand, for the out-of-sample forecasting, we compare three ARclass models with our models in forecasting future stock market returns. Table 4 shows the out-of-sample fitting MAE against ARMA, SETAR and STAR models. In general, our models outperform other three models with the improvement rate averaged around $32 \%$. Unlike the results from the in-sample fitting, the NRFM1 model exhibits weaker performance in predicting the stock return for US and Japan compared with China and India (see Table 4 and Table 5). Because out-of-sample prediction only uses the information from the past, returns in developed markets with market efficiency are unpredictable 


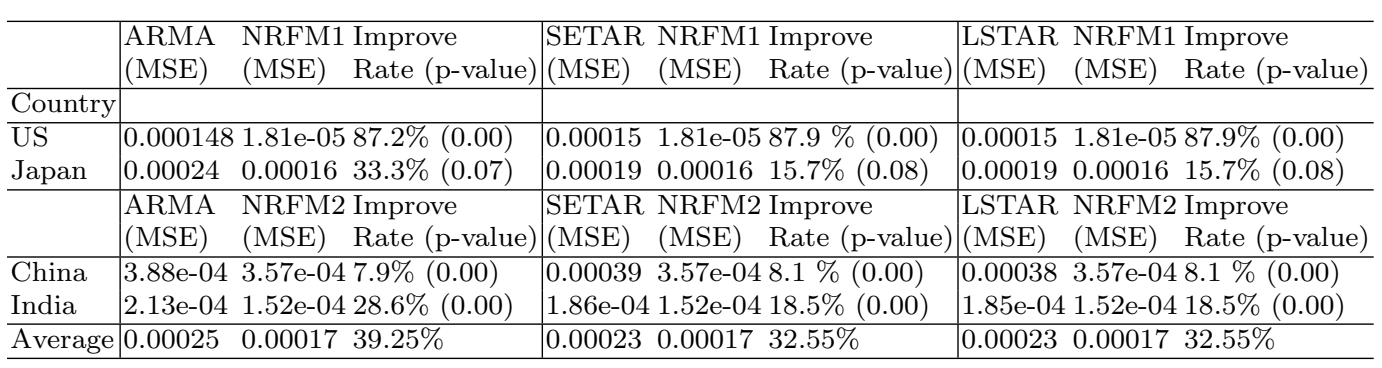

Table 3 MSE of in sample fitting of stock index returns. This table presents the in-sample fitting results of four countries' stock index return forecasting of four models using the Mean Squared Error (MSE). The p-values for statistical differences of the forecasting errors are also presented. Our models outperform all other three models. Where en $=* 10^{n}$, e.g. $e-06=* 10^{-6}$.

(Timmermann and Granger 2004). On the other side, however, emerging markets with no market efficiency might create predictable returns. Therefore, in the next section, we will propose a trading strategy based on our return prediction models. The trading strategy profit could demonstrate whether it can earn higher returns in emerging stock markets than in developed markets. From theoretical perspective, the return forecasting models should earn higher returns in emerging stock markets since returns in those markets are more predictable.

\begin{tabular}{|c|c|c|c|c|c|c|c|}
\hline & \multirow{2}{*}{\multicolumn{2}{|c|}{$\begin{array}{l}\text { ARMA NRFM1 Improve } \\
\text { (MAE) (MAE) Rate (p-value) }\end{array}$}} & \multicolumn{2}{|c|}{ SETAR NRFM1 Improve } & \multirow{2}{*}{\multicolumn{3}{|c|}{$\begin{array}{l}\text { LSTAR NRFM1 Improve } \\
\text { (MAE) (MAE) Rate (p-value) }\end{array}$}} \\
\hline & & & (MAE) (MAE) & (p-value) & & & \\
\hline \multicolumn{8}{|l|}{ Country } \\
\hline US & $0.0058 \quad 0.0045$ & $22.4 \%(0.00)$ & 0.0055 & $18.2 \%(0.00)$ & 0.0054 & 0.0045 & $18.2 \%(0.00)$ \\
\hline \multirow[t]{3}{*}{ Japan } & 0.00910 .0091 & $0.01 \%(0.30)$ & 0.0095 & $4.2 \%(0.10)$ & 0.0095 & 0.0091 & $4.2 \%(0.10)$ \\
\hline & ARMA NRFM & Improve & SETAR NRFM & $\operatorname{Imp}$ & LSTA & NRFM & $\operatorname{Imp}$ \\
\hline & (MAE) (MAE) & Rate (p-value) & (MAE) (MAE) & Rate (p-value) & (MAE) & $(\mathrm{MAE})$ & Rate (p-value) \\
\hline China & $0.0106 \quad 0.0018$ & $83.1 \%(0.00)$ & 0.0106 & $83.1 \%(0.00)$ & 0.0105 & 0.0018 & $83.1 \%(0.00)$ \\
\hline India & $0.0068 \quad 0.0052$ & $23.5 \%(0.00)$ & 0.0067 & $22.3 \%(0.00)$ & 0.0067 & 0.0052 & $22.3 \%(0.00)$ \\
\hline Average & 0.00810 .0052 & $32.25 \%$ & 0.0052 & $31.95 \%$ & 0.0081 & 0.0052 & $31.95 \%$ \\
\hline
\end{tabular}

Table 4 MAE of out-of-sample forecasting of stock index returns. This table presents the out-of-sample prediction results of four countries' stock index return forecasting of four models using the Mean Absolute Error (MAE). The p-values for statistical differences of the forecasting errors are also presented. Our models outperform all other three models.

\subsection{Robustness Check}

In order to demonstrate that our results are robust, we adopt a non-linear return forecasting model other than ARMA-family models as the benchmark model, which we denote as a High Moments Return Forecasting Model (HMRFM). High moments like skewness are vastly concerned by investors in the 


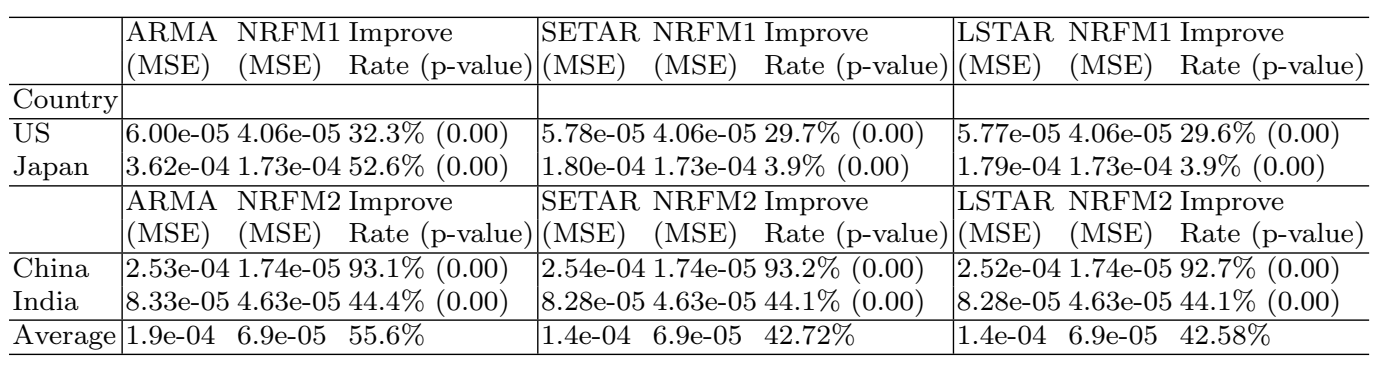

Table 5 MSE of out-of-sample forecasting of stock index returns. This table presents the out-of-sample prediction results of four countries' stock index return forecasting of four models using the Mean Squared Error (MSE). The p-values for statistical differences of the forecasting errors are also presented. Our models outperform all other three models. Where $e n=* 10^{n}$, e.g. $e-06=* 10^{-6}$.

stock market (see Kozhan et al. 2013; Kelly et al. 2014). Therefore, we adopt a HMRFM that is proposed by (Jondeau et al. 2019) as our benchmark model, which provides nonlinear relation investigation between return and high moments. The format of the model can be written as follows:

$$
r_{t}=\alpha+\beta_{1} \sigma_{t-1}^{2}+\beta_{2} s k_{t-1}+\varepsilon_{t}
$$

where $\sigma_{t}$ is the volatility of the return at time $t$ (also known as second central moment), calculated by $\sqrt{\sum_{t=1}^{n}\left(r_{t}-\overline{r_{t}}\right)^{2}}, s k_{t}$ is the skewness at time $t$ (also known as third central moment), calculated by $\sum_{t=1}^{n}\left(\frac{r_{t}-\overline{r_{t}}}{\sigma}\right)^{3}$, and $\overline{r_{t}}$ is the average return during the period.

Table 6 shows the in-sample fitting MAE and MSE of our models against HMRFM. In general, our models outperform HMRFM with the improvement rate averaged around $50 \%$ for MAE and around $90 \%$ for MSE. On the other hand, our models also surpass the HMRFM regarding the out-of-sample forecasting. Table 7 presents the out-of-sample forecasting MAE and MSE of our model against HMRFM. It can be observed that our models outperform HMRFM by around $60 \%$ for MAE and around $90 \%$ for MSE. These results have demonstrated the robustness of our models.

\section{Stock Index Futures Trading Strategy Based on the Return Forecasting Models}

In order to show different return predictability in different markets, we propose a trading strategy by trading the corresponding stock index futures based on the two return forecasting models we have developed. We aim to reveal different trading profitability in different markets by using the same trading strategy, which can be described as follows. 


\begin{tabular}{|c|c|c|c|c|c|c|}
\hline & \multicolumn{3}{|c|}{ HMRFM NRFM1 Improve } & \multicolumn{3}{|c|}{ HMRFM NRFM1 Improve } \\
\hline & (MAE) & (MAE) & Rate (p-value) & (MSE) & (MSE) & Rate (p-value) \\
\hline \multicolumn{7}{|l|}{ Country } \\
\hline$\overline{\mathrm{US}}$ & 0.016 & .0029 & $81.8 \%(0.00)$ & 0.00041 & $1.81 \mathrm{e}-0$ & $95.6 \%(0.00)$ \\
\hline \multirow[t]{2}{*}{ Japan } & 0.015 & 0.0088 & $87.3 \%(0.00)$ & 0.00046 & 0.00016 & $65.2 \%(0.00)$ \\
\hline & $\begin{array}{l}\text { HMRFM } \\
\text { (MAE) }\end{array}$ & $\begin{array}{l}\text { NRFM2 } \\
\text { (MAE) }\end{array}$ & $\begin{array}{l}\text { Improve } \\
\text { Rate (p-value) }\end{array}$ & $\begin{array}{l}\text { HMRFM } \\
\text { (MSE) }\end{array}$ & $\begin{array}{l}\text { NRFM } \\
\text { (MSE) }\end{array}$ & $\begin{array}{l}\text { Improve } \\
\text { Rate (p-value) }\end{array}$ \\
\hline mina & 0.0201 & 0.012 & $40.1 \%(0.00)$ & 0.00084 & $3.57 \mathrm{e}-0$ & $95.8 \%(0.00)$ \\
\hline India & 0.015 & 0.0092 & $38.6 \%(0.00)$ & 0.00056 & $1.52 \mathrm{e}-0$ & $97.3 \%(0.00)$ \\
\hline Average & 0.0165 & 0.0082 & $50.3 \%$ & 0.00568 & 0.00017 & $88.5 \%$ \\
\hline
\end{tabular}

Table 6 MAE and MSE for in-sample fitting of stock index returns. This table presents both Mean Absolute Error (MAE) and Mean Squared Error (MSE) in-sample fitting results of four countries' stock index return regarding the two models. The p-values for statistical differences of the forecasting errors are also presented. Our models outperform HMRFM and our results are robust. Where $e n=* 10^{n}$, e.g. $e-06=* 10^{-6}$.

\begin{tabular}{|c|c|c|c|c|c|c|}
\hline & \multicolumn{3}{|c|}{ HMRFM NRFM1 Improve } & \multirow{2}{*}{\multicolumn{3}{|c|}{\begin{tabular}{|lll} 
HMRFM NRFM1 Improve \\
$(\mathrm{MSE})$ & (MSE) & Rate (p-value) \\
\end{tabular}}} \\
\hline & (MAE) & (MAE) & Rate (p-value) & & & \\
\hline \multicolumn{7}{|l|}{ Country } \\
\hline US & 0.011 & & 59 . & 0. & 4.06 & 84 \\
\hline \multirow[t]{3}{*}{ Japan } & 0.013 & 0.0 & 30. & & 1.7 & \\
\hline & $\mathrm{HM}$ & & & 11 & $v_{1}$ & 1 \\
\hline & $(\mathrm{M}$ & $(\mathrm{M}$ & $\mathrm{Ra}$ & $(\mathrm{M}$ & (MSE) & $\mathrm{Ra}$ \\
\hline Chin & 0.01 & 0 & 87. & 0.00 & $1.74 \mathrm{e}-05$ & $96.7 \%(0$ \\
\hline Ind & 0.012 & 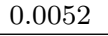 & 56.6 & 0.00028 & $4.63 \mathrm{e}-05$ & $83.5 \%(0$. \\
\hline Aver & 0.0125 & 0.0052 & $58.4 \%$ & 0.00036 & $6.9 \mathrm{e}-05$ & $90.1 \%$ \\
\hline
\end{tabular}

Table 7 MAE and MSE for out-of-sample forecasting of stock index returns. This table presents both Mean Absolute Error (MAE) and Mean Squared Error (MSE) for out-of-sample forecasting results of four countries' stock index return regarding the two models. The p-values for statistical differences of the forecasting errors are also presented. Our models outperform HMRFM and our results are robust. Where $e n=* 10^{n}$, e.g. $e-06=$ $* 10^{-6}$.

Suppose we are now at time $t$ before the market open time. We use our model to forecast the stock index return for time $t$. If the forecasted return is positive, then we long the corresponding stock index futures at its open price and we close our contract at its close price. On the other hand, if the forecasted return is negative, then we short the corresponding stock index futures at its open price and we also close our contract at its close price. At the end of time $t$, it is observable whether our strategy is successful or not. If the actual return is positive and we long the futures, then we earn the corresponding stock index futures return at time $t$. Otherwise, we lose the return of the same amount. The situation is exactly identical for the short position and negative return.

Therefore, the cumulative return of the trading strategy for each stock market is defined as:

$$
R^{T S}=\sum_{t=1}^{T} r_{t}^{T S}
$$


In particular,

$$
r_{t}^{T S}=\left|\ln \left(\frac{F_{t}^{c}}{F_{t}^{o}}\right)\right| * I\left(r_{t}^{I F} r_{t}^{F R} \geq 0\right)
$$

where $r_{t}^{T S}$ is the return from the trading strategy at time t, $F_{t}^{o}$ is the stock index futures open price at time $\mathrm{t}$ and $F_{t}^{c}$ is the stock index futures close price at time t, $r_{t}^{I F}$ is the stock index futures return at time $\mathrm{t}$ and $r_{t}^{F R}$ is the forecasted stock index futures return at time $\mathrm{t}, I(\cdot)$ is the indicator function, when $r_{t}^{I F} r_{t}^{F R} \geq 0$, then $I=1$, and $I=-1$, otherwise.

Then, we adopt the trading strategy to test our models against other four models based on the out-of-sample results (i.e. from January 1, 2012 to December 31 2017). It might be essential to point out the irrelevance of transaction cost for the trading strategy. The transaction cost of the trading strategy based on our forecasting model to compare with other AR-family models as well as the HMRFM, is trivial because all models are under similar trading mechanics, which incur the same amount of transaction cost. In other words, all models would be deducted same amount of transaction cost from their returns, which has little impact on the result. The empirical results have been presented in Table 8. From Table 8, it is clear that our model has substantial positive returns for all four countries over the 6 -year period. Our model also exhibits the additional returns compared with other three ARMA models and HMRFM. Specifically, for the NRFM1, the returns for developed countries of our model slightly outperforms other four models. On the other side, however, the returns for emerging countries of our model (NRFM2) considerably outperforms other four models. In comparison between NRFM1 and NRFM2, the returns we can earn by using the trading strategy in emerging markets are substantially higher than we can earn in developed markets. Therefore, this result aligns with theoretical prediction that returns in emerging market are more predictable, which may lead to higher returns. More importantly, this empirical results also show that the nonlinear return forecasting model has large superiority in the emerging countries, which is also consistent with the existing arguments (see Avdoulas et al. 2018).

From different trading strategies, the simple moving average (MA) trading strategy remains popular in the stock market (Fong and Yong 2005). The advantages of MA strategy involve that it can easily smooth out market noise and then follow the real market trend. The fact that financial practitioners adopt MA strategy in making buy and sell decisions in the stock market endures for decades. Therefore, it is observable that the ARMA model has a higher cumulative return compared with other two AM-family models. More importantly, information inefficiency in the market could weaken the prompt adjustment of prices responding and reflecting all public available information. From the efficient market hypothesis, information inefficiency could present in the market efficiency in either weak form or semi-strong form. As a consequence, market prices could be massively impacted by psychological factors, which shall be analyzed under an irrational theoretical framework (Menkhoff 2010). The models we built incorporate jumps in the return process to reflect 
informational surprises or news. This part helps our models to grab the discontinuity in information inefficient markets and thus provide higher returns in those markets. For the further model comparison, we present the hit ratios of all models in Table 9, which indicate the successful trading percentage of each model during the sample period. It is observable that our model has the largest hit ratio among all models. As a result, our model can be envisioned as the best performance model in both return earned and trading success.

\begin{tabular}{l|lllll}
\hline Country & \multicolumn{5}{|c}{} \\
\hline & ARMA & SETAR & LSTAR & HMRFM & NRFM1 \\
\hline US & $22.3 \%$ & $23.2 \%$ & $23.2 \%$ & $27.8 \%$ & $35.6 \%$ \\
Japan & $21.9 \%$ & $21.3 \%$ & $21.2 \%$ & $24.5 \%$ & $30.9 \%$ \\
\hline & ARMA & SETAR & LSTAR & HMRFM & NRFM2 \\
\hline China & $40.8 \%$ & $39.3 \%$ & $39.2 \%$ & $39.8 \%$ & $56.9 \%$ \\
India & $81.1 \%$ & $75.9 \%$ & $75.9 \%$ & $88.5 \%$ & $97.7 \%$ \\
\hline Average & $41.52 \%$ & $39.92 \%$ & $39.87 \%$ & $45.87 \%$ & $55.28 \%$ \\
\hline
\end{tabular}

Table 8 Cumulative returns of trading strategy based on the return forecasting models. This table presents the out-of-sample results of four countries' stock index futures returns according to the trading strategy based on the return forecasting models. Our models have significantly higher returns than all other models.

\begin{tabular}{l|lllll}
\hline Country & & & & & \\
\hline & ARMA & SETAR & LSTAR & HMRFM & NRFM1 \\
\hline US & $45.7 \%$ & $44.3 \%$ & $41.1 \%$ & $44.8 \%$ & $51.8 \%$ \\
Japan & $51.8 \%$ & $49.3 \%$ & $48.9 \%$ & $49.5 \%$ & $54.2 \%$ \\
\hline & ARMA & SETAR & LSTAR & HMRFM & NRFM2 \\
\hline China & $48.1 \%$ & $47.2 \%$ & $46.7 \%$ & $49.3 \%$ & $51.4 \%$ \\
India & $49.1 \%$ & $48.7 \%$ & $48.2 \%$ & $52.1 \%$ & $53.9 \%$ \\
\hline
\end{tabular}

Table 9 Hit ratios of trading strategy based on the return forecasting models.

\section{Conclusion}

To conclude, we have built two return forecasting models based on GP method for both developed markets and emerging markets. Our developed models have superior properties in many respects. Firstly, our model only uses lagged returns as predictors rather than filling copious variables into the model. Secondly, our models are AI based propositions, which incorporate special relations in the stock market, such as nonlinearity. Thirdly, our models distinguish developed markets from emerging markets, which restore market characteristics into our models. Empirically, we show that our models present significant improvements in return forecasting compared with AutoRegressive (AR) family models in both linear and nonlinear forms. The improvement rate is around $30 \%$ for in-sample fitting and around $40 \%$ for out-of-sample forecasting. Based 
on those precise return forecasting models, we also propose a trading strategy and the trading strategy has been verified to be highly profitable in both developed markets and emerging markets. In particular, compared with other AR-family models, our model earned $55 \%$ profitability on average while other models only earned $40 \%$. Specifically, our model earned $35 \%$ and $30 \%$ annualized return in US and Japan respectively. In contrast, other models earned $23 \%$ and $21 \%$ annualized return in US and Japan respectively. For emerging economics, our model earned $57 \%$ and $98 \%$ annualized return in China and India respectively. In contrast, other models earned $40 \%$ and $75 \%$ annualized return in China and India respectively. It is arguable that our model displays superior performance over other models with same trading strategy in the futures markets. This superior performance could be attributed to the nonlinearity capture in our model. Moreover, our models also outperform the traditional non-linear model, which illustrates the outstanding information extraction ability of the GP approach. 


\section{Bibliography}

Andersen TG, Bollerslev T (1998) Deutsche mark-dollar volatility: intraday activity patterns, macroeconomic announcements, and longer run dependencies. Journal of Finance 53(1):219-265

Ang A, Bekaert G (2006) Stock return predictability: Is it there? The Review of Financial Studies 20(3):651-707

Avdoulas C, Bekiros S, Boubaker S (2018) Evolutionary-based return forecasting with nonlinear star models: evidence from the eurozone peripheral stock markets. Annals of Operations Research 262(2):307-333, DOI $10.1007 / \mathrm{s} 10479-015-2078-\mathrm{z}$

Aydogan K, Gursoy G (2000) P/e and price-to-book ratios as predictors of stock returns in emerging equity markets. Emerging Markets Quarterly $4(4): 60-67$

Banzhaf W, Francone FD, Keller RE, Nordin P (1998) Genetic programming: an introduction: on the automatic evolution of computer programs and its applications. Morgan Kaufmann Publishers Inc., San Francisco, CA, USA

Bates DS (1996) Jumps and stochastic volatility: Exchange rate processes implicit in deutsche mark options. The Review of Financial Studies 9(1):69 107

Batten JA, Lucey BM, McGroarty F, Peat M, Urquhart A (2018) Does intraday technical trading have predictive power in precious metal markets? Journal of International Financial Markets, Institutions and Money 52:102 - 113, DOI https://doi.org/10.1016/j.intfin.2017.06.005

Bhola J, Soni S, Cheema GK (2019) Genetic algorithm based optimized leach protocol for energy efficient wireless sensor networks. Journal of Ambient Intelligence and Humanized Computing pp 1-8

Bollerslev T, Todorov V, Xu L (2015) Tail risk premia and return predictability. Journal of Financial Economics 118(1):113 - 134, DOI doi.org/10.1016/ j.jfineco.2015.02.010

Bollerslev T, Patton AJ, Quaedvlieg R (2016) Exploiting the errors: A simple approach for improved volatility forecasting. Journal of Econometrics 192(1):1-18

Cai Z, Wang Y, Wang Y (2015) Testing instability in a predictive regression model with nonstationary regressors. Econometric Theory 31(5):953980, DOI 10.1017/S0266466614000590

Campbell JY, Shiller RJ (1988) The dividend-price ratio and expectations of future dividends and discount factors. The Review of Financial Studies $1(3): 195-228$

Campbell JY, Yogo M (2006) Efficient tests of stock return predictability. Journal of Financial Economics 81(1):27 - 60, DOI doi.org/10.1016/j.jfineco. 2005.05.008

Chan WH, Maheu JM (2002) Conditional jump dynamics in stock market returns. Journal of Business \& Economic Statistics 20(3):377-389, DOI $10.1198 / 073500102288618513$ 
Chen WH, Shih JY, Wu S (2006) Comparison of support-vector machines and back propagation neural networks in forecasting the six major asian stock markets. International Journal of Electronic Finance 1(1):49-67

Chen X, Gao P (2019) Path planning and control of soccer robot based on genetic algorithm. Journal of Ambient Intelligence and Humanized Computing pp 1-10

Choudhry R, Garg K (2008) A hybrid machine learning system for stock market forecasting. World Academy of Science, Engineering and Technology 39(3):315-318

Cremers M, Weinbaum D (2010) Deviations from put-call parity and stock return predictability. Journal of Financial and Quantitative Analysis 45(2):335367, DOI 10.1017/S002210901000013X

Cremers M, Halling M, Weinbaum D (2015) Aggregate jump and volatility risk in the crosssection of stock returns. The Journal of Finance 70(2):577-614

Ding Z, Granger CW, Engle RF (1993) A long memory property of stock market returns and a new model. Journal of Empirical Finance 1(1):83 106, DOI doi.org/10.1016/0927-5398(93)90006-D

Facchini F, Digiesi S, Mossa G (2020) Optimal dry port configuration for container terminals: A non-linear model for sustainable decision making. International Journal of Production Economics 219:164-178

Fama EF (1970) Efficient capital markets: A review of theory and empirical work. The Journal of Finance 25(2):383-417

Fama EF, French KR (1988) Dividend yields and expected stock returns. Journal of Financial Economics 22(1):3 - 25, DOI doi.org/10.1016/ 0304-405X(88)90020-7

Ferrara L, Marcellino M, Mogliani M (2015) Macroeconomic forecasting during the great recession: The return of non-linearity? International Journal of Forecasting 31(3):664 - 679, DOI doi.org/10.1016/j.ijforecast.2014.11.005

Fong WM, Yong LH (2005) Chasing trends: recursive moving average trading rules and internet stocks. Journal of Empirical Finance 12(1):43 - 76, DOI doi.org/10.1016/j.jempfin.2003.07.002

Gencay R, Selcuk F (2004) Extreme value theory and value-at-risk: Relative performance in emerging markets. International Journal of Forecasting 20(2):287 - 303, DOI doi.org/10.1016/j.ijforecast.2003.09.005

Golez B, Koudijs P (2018) Four centuries of return predictability. Journal of Financial Economics 127(2):248 - 263, DOI doi.org/10.1016/j.jfineco.2017. 12.007

Golosnoy V, Hamid A, Okhrin Y (2014) The empirical similarity approach for volatility prediction. Journal of Banking \& Finance 40(Supplement C):321 - 329, DOI doi.org/10.1016/j.jbankfin.2013.12.009

Goyal A, Welch I (2003) Predicting the equity premium with dividend ratios. Management Science 49(5):639-654, DOI 10.1287/mnsc.49.5.639.15149

Hansen BE (1997) Inference in tar models. Studies in Nonlinear Dynamics \& Econometrics 2(1)

Hansen BE (2000) Sample splitting and threshold estimation. Econometrica 68(3):575-603 
Hirsh H, Banzhaf W, Koza JR, Ryan C, Spector L, Jacob C (2000) Genetic programming. IEEE Intelligent Systems 15(3):74-84

Hurn AS, Silvennoinen A, Tersvirta T (2016) A smooth transition logit model of the effects of deregulation in the electricity market. Journal of Applied Econometrics 31(4):707-733

Jiang F, Tong G, Song G (2019) Technical analysis profitability without data snooping bias: Evidence from chinese stock market. International Review of Finance 19(1):191-206

Jiang X, Lee BS (2007) Stock returns, dividend yield, and book-to-market ratio. Journal of Banking \& Finance 31(2):455 - 475, DOI doi.org/10.1016/ j.jbankfin.2006.07.012

Jondeau E, Zhang Q, Zhu X (2019) Average skewness matters. Journal of Financial Economics 134(1):29-47

Kelly B, Jiang H (2014) Tail risk and asset prices. The Review of Financial Studies 27(10):2841-2871

Kim HY, Mei JP (2001) What makes the stock market jump? an analysis of political risk on hong kong stock returns. Journal of International Money and Finance 20(7):1003 - 1016, DOI doi.org/10.1016/S0261-5606(01)00035-3

Kim JH, Shamsuddin A (2008) Are asian stock markets efficient? evidence from new multiple variance ratio tests. Journal of Empirical Finance 15(3):518 - 532, DOI doi.org/10.1016/j.jempfin.2007.07.001

Kou SG (2002) A jump-diffusion model for option pricing. Management Science 48(8):1086-1101

Kozhan R, Neuberger A, Schneider P (2013) The skew risk premium in the equity index market. The Review of Financial Studies 26(9):2174-2203

Lamont O (1998) Earnings and expected returns. The Journal of Finance 53(5):1563-1587

Lee BS, Paek M, Ha Y, Ko K (2015) The dynamics of market volatility, market return, and equity fund flow: International evidence. International Review of Economics \& Finance 35:214 - 227, DOI doi.org/10.1016/j.iref.2014.10.001

Lettau M, Ludvigson S (2001) Consumption, aggregate wealth, and expected stock returns. The Journal of Finance 56(3):815-849

Liu R, Yang J, Ruan C (2019) Expected stock return and mixed frequency variance risk premium data. Journal of Ambient Intelligence and Humanized Computing pp $1-12$

Manahov V, Hudson R, Hoque H (2015) Return predictability and the wisdom of crowds: Genetic programming trading algorithms, the marginal trader hypothesis and the hayek hypothesis. Journal of International Financial Markets, Institutions and Money 37(Supplement C):85 - 98, DOI doi.org/10.1016/j.intfin.2015.02.009

Marcellino M, Stock JH, Watson MW (2006) A comparison of direct and iterated multistep ar methods for forecasting macroeconomic time series. Journal of Econometrics 135(1):499 - 526, DOI doi.org/10.1016/j.jeconom. 2005.07.020

Menkhoff L (2010) The use of technical analysis by fund managers: International evidence. Journal of Banking \& Finance 34(11):2573 - 2586, DOI 
doi.org/10.1016/j.jbankfin.2010.04.014

Nelson CR, Kim MJ (1993) Predictable stock returns: The role of small sample bias. The Journal of Finance 48(2):641-661

Park CH, Irwin SH (2007) What do we know about the profitability of technical analysis? Journal of Economic Surveys 21(4):786-826

Paye BS, Timmermann A (2006) Instability of return prediction models. Journal of Empirical Finance 13(3):274 - 315, DOI doi.org/10.1016/j.jempfin. 2005.11.001

Pimenta A, Nametala CAL, Guimarães FG, Carrano EG (2017) An automated investing method for stock market based on multiobjective genetic programming. Computational Economics DOI 10.1007/s10614-017-9665-9

Poli R, Langdon WB, McPhee NF (2008) A Field Guide to Genetic Programming. Lulu Enterprises, UK Ltd

Pong S, Shackleton MB, Taylor SJ, Xu X (2004) Forecasting currency volatility: A comparison of implied volatilities and ar(fi)ma models. Journal of Banking \& Finance 28(10):2541 - 2563, DOI doi.org/10.1016/j.jbankfin. 2003.10.015

Scheinkman JA, LeBaron B (1989) Nonlinear dynamics and stock returns. The Journal of Business 62(3):311-337

Shoba S, Rajavel R (2020) A new genetic algorithm based fusion scheme in monaural casa system to improve the performance of the speech. Journal of Ambient Intelligence and Humanized Computing 11:433-446

Terasvirta T (1994) Specification, estimation, and evaluation of smooth transition autoregressive models. Journal of the American Statistical Association 89(425):208-218, DOI 10.1080/01621459.1994.10476462

Timmermann A, Granger CW (2004) Efficient market hypothesis and forecasting. International Journal of Forecasting 20(1):15 - 27, DOI doi.org/10. 1016/S0169-2070(03)00012-8

Tong H (1978) On a threshold model

Tong H (1990) Non-linear time series: a dynamical system approach. Oxford University Press

Welch I, Goyal A (2007) A comprehensive look at the empirical performance of equity premium prediction. The Review of Financial Studies 21(4):14551508

Zhao J, Xiang Y, Yang S, Zhang D, Yu P (2019) The control of the movement of intelligent car based on self-adaptive non-linear algorithm. Journal of Ambient Intelligence and Humanized Computing 10(11):4595-4602 